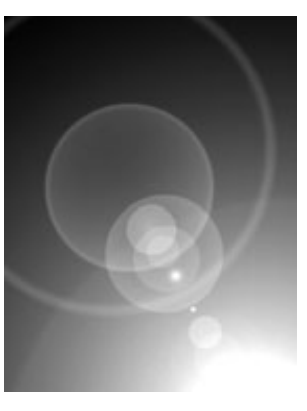

OR I G I NAL ARTICLE

\title{
Caring for children with learning disabilities: an exploratory study of parental strain and coping
}

Kate Kenny and Sinéad McGilloway, Department of Psychology, National University of Ireland at Maynooth, Maynooth, County Kildare, Ireland (E-mail: sinead.mcgilloway@nuim.ie)

Accessible summary

\section{Summary}

- Caring for a child with learning disability can be both a rewarding and difficult experience, but little is known about the experience of parents who provide care for children with learning disabilities in Ireland.

- This study involved 32 parents of children $(<16)$ with learning disabilities living in the Greater Dublin area.

- The research investigated the kind of tasks involved in caring for a child with a learning disability, the extent and nature of any difficult behaviours with which parents must cope as well as parents' attitudes towards caring. The use and availability of support services and how parents cope with their caring role were also explored.

- This research is important to people with learning disabilities and their carers because it tells us about the practical, emotional and psychological benefits and drawbacks of caring for a child with learning disability and the importance of having appropriate and effective support services for both parents and their children.

Despite recurring concerns about the role and appropriate support of informal carers, little is known about the parental experience of caring for children with learning disabilities in Ireland. This study describes and analyses the nature and consequences of care and coping among parents of children $(<16)$ with learning disabilities living in the Greater Dublin area. Participants $(n=32)$ completed the Caregiver Strain Questionnaire and an adapted version of the Carers Questionnaire which assessed: care tasks/behavioural difficulties; caregiver attitudes; service provision; and coping strategies. Qualitative analysis examined factors affecting carers' ability to cope. Participants showed high levels of objective and subjective caregiver strain and most were receiving inadequate support. However, parents employed a range of strategies to help them cope more effectively. The qualitative data highlighted the difficulties and rewards of caregiving and the inadequacies of current service provision.

Keywords Caregiver burden, children, coping, informal carers, intellectual disability, parents 


\section{Introduction}

The birth of a child with a learning disability often has a profound effect on the family, requiring one or more family members (usually the parent(s)) to adopt the role of 'carer'. Traditionally, research on caregiving for children and young people with disabilities has highlighted the stresses or 'burden' imposed on parents by the many, and often complex, demands of caregiving (Brannan et al. 1997; Egan \& Walsh 2001; Hassall et al. 2005; McGilloway et al. 1995; Saloviita et al. 2003; Shearn \& Todd 2000). Pearlin et al. (1990) describe several sources of caregiver strain including family conflict arising from issues around impairment, financial strain and a constricted social and recreational life. Other potential stressors include: restricted access to services (Redmond \& Richardson 2003); uncertainty surrounding the child's future (Todd et al. 1993; Walsh et al. 1993); and lack of formal and informal support (Egan \& Walsh 2001). Numerous studies have also shown that similar stressors give rise to widely differing levels of caregiver strain (Ferguson 2001; Grant \& Whittell 2000; Hassall \& Rose 2005).

Brannan et al. (1997) define caregiver strain as the demands, responsibilities, difficulties, and negative psychic consequences of caring for relatives with special needs'. This may be subdivided into objective strain which describes the observable occurrences and events arising from the child's problems/behaviour; and subjective strain which refers to the emotional strain of caring (Brannan et al. 1997). The difficulties of managing the care recipient contribute to objective caregiver strain whilst subjective strain is more strongly related to caregiver characteristics and comprises internalized (e.g. worry, sadness) and externalized subjective strain (e.g. anger or resentment towards the child or the behaviour). Brannan et al. suggest that families caring for children with disabilities may experience higher levels of subjective strain and lower levels of objective strain when compared with carers of adults.

Recent research in this area has focused on the role of coping strategies in mediating the relationship between stressful events and adaptive outcomes including resilience (e.g. Grant \& Ramcharan 2001; Grant et al. 1998; Hastings \& Taunt 2002; Kelso et al. 2005; Sharry 1999; Taanila et al. 2002). Specifically, coping strategies describe the processes and behaviours employed by carers to manage stressful situations (Taanila et al. 2002). Burr \& Klein (1994) provide a useful conceptual framework within which a large number of coping strategies relating to familial stress have been described and classified. This has proved effective in examining the coping strategies employed by parents of children with intellectual disabilities (Taanila et al. 2002). Adaptive forms of coping include: maintaining an optimistic attitude and positive perceptions of the child; seeking information about the child's condition; and developing family cohesion. Seeking informal social support and having access to formal support services are widely acknowledged to be the most effective coping strategies (e.g. Egan \& Walsh 2001; Redmond \& Richardson 2003; Solomon et al. 2001; Taanila et al. 2002; White \& Hastings 2004).

Much previous research has examined either caregiver strain or the coping processes employed mainly by carers of adults or caregivers of children across a relatively narrow spectrum of disability (e.g. Carr \& O'Reilly 1996; Egan \& Walsh 2001; McGilloway et al. 1995; Redmond \& Richardson 2003; Sharry 1999). The present study attempts to examine both perspectives in order to better understand the experience of Irish parents caring for children $(<16)$ with a wide range of intellectual disabilities. Additionally, there are few qualitative findings in this area (e.g. Kelso et al. 2005) whilst little research of this kind has been conducted in Ireland. Countries such as the UK, unlike Ireland, have a long tradition of providing formal support for individuals with learning disabilities and their carers. Recent figures from the National Intellectual Disability Database for Ireland (Barron \& Mulvaney 2004) indicate that there are 25,416 children and adults with learning disabilities in Ireland, approximately one-third of whom are aged 19 years or under. (Prevalence rates vary from 4.06 per 1000 for those aged $\leq 4$ years to 9.93 per 1000 for 15- to 19-year-olds.) Arguably, the implementation of a new Disability Bill (Disability Federation of Ireland 2004) in Ireland goes some way towards addressing concerns around the accessibility, organization and delivery of services for individuals with learning disabilities and their families. However, many continue to express misgivings about the new Bill. Furthermore, policy and service development ought to be sensitive to the views and needs of service users and their caregivers, but it is not clear to what extent this is currently the case in Ireland. The aim of this exploratory study was to contribute to this public debate by: (1) assessing levels of caregiver strain; (2) describing the practical day-to-day aspects of caring and the extent and nature of formal and informal support; and (3) exploring the coping strategies employed by carers including a qualitative assessment of factors affecting coping ability.

\section{Method}

\section{Participants}

A convenience sample of 32 participants aged $28-57$ years (mean $=44, \mathrm{SD}=7.08$ ) was recruited from the friends and acquaintances of the first author (KK) who is also the mother of a 16-year-old girl with a moderate learning disability. Each participant was the mother/father of a child $(<18)$ with an intellectual disability. All participants were living in the Greater Dublin area and most (24/32) were members of several different Parent Support Groups. 


\section{Measures}

The Caregiver Strain Questionnaire (CGSQ) (Brannan et al. 1997; Kang et al. 2005), is a 21-item, self-report instrument which has been widely used in the research literature to assess both objective (10 items) and subjective dimensions (11 items) of caregiver strain. The CGSQ (previously referred to as the Burden of Care Questionnaire) was designed for use with families of children with mental, emotional and behavioural problems, but includes an assessment of all of the key areas identified in caregiver research. Therefore, it is highly relevant to carers of children with intellectual disabilities, most of whom have significant behavioural and emotional problems. Following a brief pilot study, it was considered to be entirely suitable for use in the present study with the exception of one minor modification whereby the word 'difficulties' in each question was substituted for 'problems' as this was considered to be more appropriate to a child with a learning disability.

The areas assessed (relating to the previous 6 months) include: disruption of family life and relationships; time demands; effects on the mental/physical health of family members; financial strain; sacrifice; disruption of social/ community life; worry and guilt; fatigue and strain; and embarrassment. For example, item 1 is 'Interruption of personal time resulting from your child's problems'. Responses are scored on a five-point scale scored from 1 ('not at all' a problem) to 5 ('very much' a problem) to yield total scores from 21 (low strain) to 105 (high strain). Brannan et al. conducted a series of analyses to indicate good reliability and validity of the CGSQ and to support the existence of the various dimensions of caregiving described earlier (e.g. Brannan \& Heflinger 2001; Brannan et al. 1997). In the present study, the CGSQ and its three subscales demonstrated very good internal consistency (i.e. Cronbach's $\alpha=0.92$ for the objective strain subscale; 0.74 for the externalized subjective strain subscale; and 0.86 for the internalized subjective strain subscale; 0.93 for the entire scale). Subscale scores were calculated as the mean of the items within each subscale whilst the global strain score was the mean of all of the items.

The second questionnaire comprised an adapted version of the Carers Questionnaire (McGilloway et al. 1995). This was originally designed for carers of adults with learning disabilities and includes four sections which were considered highly relevant, with some modification, to carers of children with learning disabilities. The first section elicits sociodemographic and background information (e.g. parental employment, child's diagnosis) whilst the second section focuses on the personal experience and practical aspects of providing care. It comprises: (1) two open-ended subsections in which respondents describe the positive and negative aspects of caring, how they cope and ways in which they think caring could be made easier; and (2) two sets of structured questions on respondents' ability to manage their child's social functioning and behavioural problems. Here, respondents are required to indicate, on a four-point Likert scale, their level of difficulty in providing practical assistance with 13 everyday tasks (e.g. mixing with others) and 19 problem behaviours (e.g. mood swings). Some of these items were modified or deleted for purposes of this study. Each question is scored from 1 ('no help given') to 4 ('difficult to manage') to generate total scores ranging from 13 to 56 on social functioning and 19 to 76 on problem behaviour. Higher scores indicate greater difficulty. Section 3 assesses respondents' satisfaction with a range of services whilst section 4 contains 10 questions based around Burr and Klein's coping framework and devised specifically to ascertain the extent to which carers employ coping strategies. Typically, interviews lasted at least 1 hour, approximately half of which was spent on the open-ended sections.

\section{Ethical and good practice statement}

The study was conducted in accordance with the British Psychological Society Code of Conduct. All participants gave their written informed consent and were assured of confidentiality. They were also informed that they could withdraw from the study (or withdraw their data) at any time. All questions and concerns were addressed by the research interviewer (KK), after which each participant was offered a summary of the findings in due course. The findings have also been disseminated to professionals at national and international conferences.

\section{Results}

Participants were predominantly female (24/32, 75\%), married and from a wide range of socioeconomic backgrounds. Half were in employment outside the home whilst most of the remainder had ceased employment in order to care for their child. The care recipients were aged between 2 and 17 years $($ mean $=11$ years, $S D=3.94)$, most were male $(19 / 32)$ and all had other siblings. Approximately one in five $(7 / 32)$ had a diagnosis (DSM-IV-TR) of mild learning disability (IQ score 50-70) with more than one in ten $(4 / 32$ ) classified as severe (IQ score 20-34). The remainder (19/32) were diagnosed as having a moderate level of disability (IQ score 35-49). (Two children did not receive a formal diagnosis.) Almost half of the children (14/32, 44\%) had concurrent physical disabilities.

\section{Caregiver strain}

CGSQ scores ranged from 25 to 77 (mean $=45$; SD $=14.82$ ). Almost three-quarters (23/32) were worried about their child's future, nearly one-third (10/32) felt tired or strained 
as a result of their child's difficulties, whilst more than one in five $(7 / 32)$ indicated that the child's difficulties had taken a toll on their family. A global measure of caregiver strain was calculated using the mean of all items (2.1) together with measures of objective (2.0) and subjective strain. The two subjective strain measures indicated that participants reported higher levels of internalized (2.9) than externalized strain (1.6) and, therefore, that their negative feelings about their caring role imposed the greatest strain on their lives. The predominance of females precluded the possibility of examining gender differences whilst an analysis by age indicated only a marginal difference in total CGSQ scores $(P=0.049, t(29)=2.05)$ between parents aged over and under 45 (the group was almost equally divided between the two). There were no differences $(P>0.05)$ between parents of children with and without physical disabilities.

\section{Practical aspects of caring and support received}

Participants reported most difficulty in helping their children to manage money, shopping and interacting with others. Total scores on social functioning ranged from 22 to 47 (mean $=36 ; \mathrm{SD}=8.34$ ). Problem behaviours such as poor concentration, restlessness and mood swings were reported by participants to be the most difficult to manage and total scores on this scale ranged from 26 to 87 $($ mean $=50 ; \mathrm{SD}=15.85)$. On balance, the distribution of scores indicated that participants tend to experience marginally more difficulty with the practical or social care tasks than with managing problem behaviour. The scores on both scales also showed moderate, positive, statistically significant correlations with total caregiver strain scores $(r=0.54$ and 0.56 , respectively, $P<0.01$ ). Virtually all participants appeared to derive considerable support from their spouses/partners, their other children, friends and other parents (Table 1). A substantial proportion was in contact with a social worker, although only one-third were satisfied with the nature and amount of support received. Similarly, most of the smaller number of people who were seeing a community nurse or psychologist were dissatisfied with the support provided (Table 1). The overall picture emerging from these findings is one of relatively low service provision compounded by generally low levels of satisfaction with formal support.

\section{Coping}

More than two-thirds (21/32) of the parents sought professional support. Thirteen parents were given information at the time of their child's diagnosis, but only three were satisfied with the information provided whilst more than half $(19 / 32)$ felt that they had insufficient information about their child's condition as he/she was growing older. Importantly, all but three participants took part in (and
Table 1 Support received and satisfaction with support

\begin{tabular}{lrr}
\hline Type of support & No. (\%) received & No. (\%) satisfied \\
\hline Formal support & & \\
$\quad$ Social worker & $14(44)$ & $5(36)$ \\
Psychologist & $11(34)$ & $2(18)$ \\
General Practitioner & $22(29)$ & $14(64)$ \\
Community nurse & $8(25)$ & $3(38)$ \\
Domiciliary services & $5(16)$ & $1(20)$ \\
Informal support & & \\
Spouse/partner & $31(97)$ & $30(97)$ \\
Other children & $30(94)$ & $28(94)$ \\
School & $30(94)$ & $18(60)$ \\
Friends & $25(85)$ & $16(64)$ \\
Other parents & $27(84)$ & $20(74)$ \\
Respite care & $15(47)$ & $11(73)$ \\
Carer support group & $5(16)$ & $1(20)$ \\
Voluntary organization & $8(16)$ & $4(50)$ \\
Welfare benefits & $31(97)$ & $8(80)$ \\
Other & $10(31)$ & \\
\hline
\end{tabular}

valued) regular, or occasional family activities and most (26/ 32) also had hobbies or engaged in activities on their own. More than two-thirds (22/32) felt that their child would not be able to cope with his/her life and a similar proportion indicated that appropriate support systems would be unavailable for them in the future. Twenty participants expressed concern that there would be no one to look after their child when they were old or had passed on, whilst 13 refused to think about the future. Almost half (14/32) reported that their lives were restricted by their caring role, although all but three felt satisfied with their life at present.

\section{Qualitative findings}

The responses to the open-ended questions were extracted from the questionnaire, combined and examined as an integrated whole using thematic analysis (see Hayes 2000) to identify key themes and messages relating to parental coping. Parents spoke about profound changes in their lives as a result of caring for their child, but such changes often brought fulfilment to their lives and this appeared to help them cope reasonably well. For example, many parents expressed a deep personal bond with their child which they felt was unlike any other. They also described the joy that their child had brought to them and to others:

She seems to bring out the very best in people, even the hardest and the 'coolest'! (Parent of 2-year old without diagnosis).

My daughter is not a burden. She is a positive influence on my life. (Parent of 15-year-old with moderate LD).

A major theme related to the positive changes which caregiving had brought to participants' lives which, in turn, 
had helped them to cope better. For example, the collective findings suggest that they became less judgemental, materialistic and selfish than before their child was born, and more confident, open and honest, and more appreciative of the 'little things in life'. For example, four respondents felt that they had become more optimistic and that they laughed more. Others pointed to their realization of how much they could do to help their children reach their potential, thereby exploiting their own talents. This is illustrated well by the comments of one father who described caring for his daughter as:

...a true test of my capacity - to refuse to accept any problem without finding a solution. (Parent of 2-yearold with mild LD).

One mother felt that she had become more creative and imaginative when teaching new tasks and another felt empowered to speak out on her child's behalf. Eight parents reported meeting new friends as a result of caring for their child:

It has introduced me to a world of people I may never have had the privilege of knowing. (Parent 16 of 9-yearold with mild LD).

However, almost one-third of the participants commented at length on those factors which affected their ability to cope and which made caring a potentially negative experience. This is illustrated most poignantly by the following:

While looking out for our son all the time is a constant strain, there is an intangible satisfaction derived from feeling constantly involved in his progress, welfare and prospects. This, however, is clouded by the realization that we cannot look out for him forever as we get older.

(Parent of 17-year-old with moderate LD).

A key theme revolved around societal and governmental attitudes towards young people with learning disabilities. For example, eight parents expressed frustration and disappointment at the general lack of public tolerance or understanding:

I do not feel that society in general, as personified by government policies, values my son, or can conceive that he could have any valuable part to play in society (Parent of 17-year-old with moderate LD).

The third theme related to the perceived inadequacy of formal services. Four parents criticized the lack of partnership between government departments in planning interventions and the lack of funding to provide appropriate and effective services. One mother summed it up:

Trying to cope with inadequate health care resources is far more stressful and frustrating than trying to cope with any aspect of my son's special needs The hardest thing is having to fight so hard for the resources he needs - the boxing gloves can never come off! (Parent of 6-year-old with moderate LD).

Some parents highlighted a need for more teacher training in special needs and in providing more one-toone classroom assistance, whilst almost one-third suggested that their child required well supervised and appropriate youth activities. Four parents expressed a need for respite care whilst a substantial proportion required access to speech therapy and/or occupational or physiotherapy services. Improved communication with, and empathy from, medical staff were identified as important in helping parents to cope better and gain greater access to essential services/equipment:

...in our experience, the professionals still operate out of a medical model, i.e. they know best and don't take parents', and indeed clients' views into account when planning services and/or interventions. (Parent of 10-year-old with severe LD).

Parents also felt that automatic access to entitlements such as Domiciliary Care Allowance and medical cards would be beneficial. A quarter of respondents reported that access to home support services would also enhance their lives.

A final sub-theme related to the amount and quality of information provided to parents. Almost two-thirds (19/32) of participants felt that more information was essential, not only at initial diagnosis, but also to help them and their child through the different stages of their lives. More information from, and better communication with, schools were also identified as a means of enhancing parents' lives and improving their coping ability. One mother indicated a need for better:

...education of medical staff and population at large who have preconceived and largely wrong ideas of what my child is capable of. This also includes educators who at times impose limitations on my child's ability that I would never impose. (Parent of 9-year-old with mild LD).

\section{Discussion}

This study examined the experiences of parental carers of children with learning disabilities by assessing caregiver strain and aspects of coping. The study is limited by a relatively small sample size and a predominance of female participants. Additionally, participants were selected from friends and acquaintances of the first author (KK). However, there was no evidence that this had unduly influenced data collection or analysis and the findings from both the quantitative and qualitative parts of the study converge to suggest that there was no threat to internal validity due to 
familiarity with the researcher. The results are also in line with previous research. Furthermore, all participants welcomed the opportunity to talk openly and honestly with someone with whom they were reasonably familiar and with whom they felt comfortable. There was also a sufficiently long time lag between data collection and analysis to eliminate the possibility of identifying any of the parents from the responses they provided. Despite the above limitations, the study provides some interesting and informative insights into the extent and nature of caregiver strain and coping amongst parental carers of children with learning disabilities in Ireland.

Whilst the CGSQ has been used and validated in over 25 studies since 1997, no norms or clinical cut-off scores are as yet available. However, a summary of mean scores for carers of a range of American young people receiving treatment for emotional and behavioural disturbance and substance abuse is available (the six sample sizes range from 121 to 6388) (Brannan, personal communication, July 2005). Therefore, whilst no specific like-with-like comparisons can be made, it is interesting to note that the pattern of mean scores obtained in our study was in line with those seen across all of the American subgroups; that is, internalized subjective strain was highest overall, followed by objective and then externalized subjective strain. The higher levels of internalized subjective strain in our study support previous findings indicating that carers of children with learning disabilities - when compared with carers of adults - tend to experience less strain from problem behaviour and more from how they feel about the caring experience (Brannan et al. 1997; McGilloway et al. 1995).

The moderate correlations between the CGSQ and the CQ scores suggest that both measures appear to be assessing similar aspects of strain relevant to this population of carers and that social and problem behaviours form an intrinsic part of caring for a child with a learning disability. However, participants alluded relatively infrequently, in the qualitative data, to the practical demands of caring, despite the fact that substantial proportions had difficulties in managing some everyday 'social' tasks and, to a lesser extent, problem behaviours (both of which were also associated with higher levels of overall strain). Instead, participants spoke often (and poignantly) about the emotional aspects of parenting a child with a learning disability whilst acknowledging the need for effective and appropriate service provision.

The qualitative findings further suggest that there were some individuals who were suffering more than others, although many appear to have benefited from the 'buffering' effect of the support provided by their spouses/ partners and families. Family cohesion togetherness has been identified in recent research as an important coping mechanism for families (Olssen \& Hwang 2002; White \& Hastings 2004), although another study on Irish mothers of, albeit adult, children with intellectual disabilities, reported high levels of social isolation (Seltzer et al. 1995). However, the fact that more than three-quarters of the participants in the current study were receiving support from other parents may have been beneficial, as building relationships with other carers has been found to be a key indicator of coping ability (Sharry 1999; Solomon et al. 2001). Nonetheless, participants were clearly exposed to a range of well known potential stressors such as employment outside the home, difficulties in dealing with social and practical care tasks, uncertainty surrounding the future care of their child and feelings of restriction on their everyday lives (e.g. Pearlin et al. 1990; Shearn \& Todd 2000; Todd et al. 1993).

Participants employed both emotion- and problemfocused coping strategies to help them manage their caring role (Folkman et al., 2000). Most appeared to have a realistic outlook of their child's disability and sought information about their condition, both of which have been shown to be effective coping strategies (Sharry 1991; Taanila et al. 2002). However, the lack of appropriate information for parents from the initial diagnosis and beyond, is alarming and something that should be tackled at grass-roots level. This finding is consistent with Beresford (1994) and Redmond \& Richardson (2003) who reported that services for children and their families were perceived as inadequate or satisfactory. [Both of the above studies provide good comparators for the present study in terms of sample size ( $n=20$ and 17 respectively) and overall methodology.] The intrinsic importance to positive parental coping of understanding the essential nature of a diagnosis, its prognosis and implications cannot be underestimated. Further forms of adaptive coping noted in previous research (Burr \& Klein 1994; Grant \& Ramcharan 2001; Hastings et al. 2002; Todd et al. 1993) were also illustrated in our data. These include caregivers' positive perceptions of their child and his/her impact on the family, their regular engagement in hobbies/ pastimes and the largely favourable perceptions of their own life situation. For example, Beresford (1994) was one of the first to describe the positive aspects of caring for a severely disabled child whereby parents, though sometimes under considerable stress, were motivated by, and derived pleasure from, their relationship with their child.

Similarly, the current study shows, in line with the small, but growing body of work conducted elsewhere (e.g. Beresford 1994; Grant et al. 1998; Hastings et al. 2002; Heller \& Factor 1993) that there can be positive and potentially rewarding aspects of providing care for a child with a learning disability irrespective of class boundaries, and the type and degree of the child's disability. The predominantly negative conceptualization of 'caregiver burden' implies that parents view their caring role pessimistically (or, at the very least, that others view it as such). However, our data show, in line with the above studies, that this is not necessarily the case despite the lack of support services for 
carers of children with learning disabilities in Ireland. Whilst there was clear evidence of caregiver strain, most participants were satisfied with their lives, many employed effective coping strategies and almost all had realistic expectations for their children and their future. The gratifying aspects of caring identified from the qualitative data included a range of inter- and intra-personal dimensions (as described by Nolan et al. 1996) such as 'bonding' with the child (interpersonal) and deriving a sense of personal fulfilment, growing creativity and increasing selflessness (intra-personal). These illustrate that the caring relationship can be mutually beneficial to both the parents and the child and a source of considerable satisfaction to the carer, despite the lack of appropriate and responsive support services and personal difficulties which clearly form only part of the picture.

It was not possible, within the scope of this study, to examine the nature of the relationship between coping strategies and caregiver strain. Therefore, it would be useful to undertake a longitudinal study in Ireland in order to assess (and promote) coping strategies amongst parental carers of children with learning disabilities using, for example, the recently developed Carers' Assessment of Managing Index (CAMI) (Grant \& Whittell, 2000). Clearly, such research would inform the development of appropriate family-centred interventions and would also have important implications in terms of improving the overall quality of life of children.

The absence of any emphatic age-related differences in total CGSQ scores - in conjunction with some of the qualitative findings - tend to support Todd et al.'s (1993) assertion that parents may see the continuation of their active parenting as providing a purpose in life and a positively valued social role into old age. This must be balanced, though, by parents' concerns about their child's future and the lack of effective services and/or inappropriate use of existing services, both of which may have a detrimental impact on overall coping ability (e.g. Redmond \& Richardson 2003; Taanila et al. 2002.).

It is worth noting that Ireland tends to be characterized, traditionally, by strong societal and particularly religious norms which may lead to a misplaced stoicism and lower levels of parental help-seeking. Nonetheless, our findings suggest generally low levels of satisfaction with formal services. For example, physiotherapy and speech and occupational therapy services were mostly absent, whilst there was little or no proper support from community nursing, psychologists (of which there is a shortage in Ireland), or domiciliary and respite services. Informal support such as that provided by local voluntary organizations was also lacking. Likewise, two other Irish studies (Redmond \& Richardson 2003; Walsh et al. 1993) have identified the lack of formal services as an important factor in the well being of parental carers of both adults and children with learning disabilities. Reassuringly however, residential and day care services appear to have improved since 1997 due to the implementation of the National Intellectual Disability Database in Ireland. This provides a systematic means of monitoring and planning the utilization of, and need for, such services. However, the impact on carers (if any) of improvements in day care services is not known. Unfortunately, it would appear that much more is required for carers despite dramatic improvements in the Irish economy and increased recent investment in such services. Arguably, Ireland's relatively recent hosting of the World Special Olympics has gone some way towards changing societal attitudes and perceptions. However, considerable work is needed to translate this into increased funding and practical 'on the ground' help and support for parents who must cope with the demands of providing care for a child with a learning disability.

\section{Acknowledgements}

The authors would like to thank all the parents who participated in this study.

\section{References}

Barron S. \& Mulvaney F. (2004) Annual report of the National Intellectual Disability Database Committee 2004. Dublin, Ireland, Health Research Board.

Beresford B. (1994) Caring for a severely disabled child. York, Social Care Research Findings (No. 54), Joseph Rowntree Trust.

Brannan A.M. \& Heflinger C.A. (2001) Distinguishing caregiver strain from psychological distress: modelling the relationship between child, family and caregiver variables. J Child Fam Stud, 10: 405-18.

Brannan A.B., Heflinger C.A. \& Bickman L. (1997) The caregiver strain questionnaire: Measuring the impact on the family of living with a child with serious emotional disturbance. J Emotion Behav Disord, 5: 212-22.

Burr W.R. \& Klein S.R. (1994) Reexamining family stress. New theory and research. London, Sage Publications.

Carr A. \& O'Reilly M. (1996) Service needs of carers for people with intellectual disabilities: Profiles of high-need and low-need groups. Ir J Psychol, 17: 48-59.

Disability Federation of Ireland (2004) Submission to the Joint Committee on Justice, Equality, Defence and Women's Rights on the National Disability Strategy, Dublin, Ireland.

Egan J. \& Walsh P.N. (2001) Sources of stress among adult siblings of Irish people with intellectual disability. Ir J Psychol, 22: 28-38.

Ferguson P.M. (2001) Mapping the family: disability studies and the exploration of parental response to disability. In: Albrecht G.L., Seelman K.D., Bury M., editors. Handbook of disability studies.

Thousand Oaks, CA, Sage Publications.

Folkman S, Lazarus R.S., Dunkel-Schetter C., DeLongis A. \& Gruen R.J. (2000) The dynamics of a stressful encounter. In: Higgins E.T., 
Kruglonski AW., editors. Motivational science: social and personality perspectives. Philadelphia, PA, Psychology Press: 111-27.

Grant G. \& Ramcharan P. (2001) Views and experiences of people with intellectual disabilities and their families. (2) The family perspective. J Appl Res Intellect Disabil, 14: 364-89.

Grant G., Ramcharan P., McGrath M., Nolan M. \& Keady J. (1998) Rewards and gratifications among family caregivers: towards a refined model of caring and coping. J Intellect Disabil Res, 42: $58-71$.

Grant G. \& Whittell B. (2000) Differentiated coping strategies in families with children or adults with intellectual disabilities: the relevance of gender, family coposition and the life span. J Appl Res Intellect Disabil, 13: 256-75.

Hassall R. \& Rose J. (2005) Parental cognitions and adaptation to the demands of caring for a child with an intellectual disability: a review of the literature and implications for clinical interventions. Behav Cogn Psychother, 33: 71-88.

Hassall R., Rose J. \& McDonald J. (2005) Parenting stress in mothers of children with an intellectual disability: the effects of parental cognitions in relation to child characteristics and family support. J Intellect Disabil Res, 49: 405-18.

Hastings R.P. \& Taunt H.M. (2002) Positive perceptions in families of children with developmental disabilities. Am J Ment Retard, 107: 166-27.

Hastings R.P., Allen R., McDermott K. \& Still D. (2002) Factors related to positive perceptions in mothers of children with intellectual disabilities. J Appl Res Intellect Disabil, 15: 269.

Hayes N. (2000) Doing psychological research: gathering and analysing data. Buckingham, Open University Press.

Heller T. \& Factor A. (1993) Aging family caregivers: support resources and changes in burden and placement desire. Am J Ment Retard, 98: 417-26.

Kang E.J., Brannan A.M. \& Heflinger C.A. (2005) Racial differences in reports of caregiver strain among caregivers of children with emotional and behavioral problems. J Child Fam Stud, 14: 43-56.

Kelso T., French D. \& Fernandez M. (2005) Stress and coping in primary caregivers of children with a disability: a qualitative study using eh Lazarus and Folkman Process Model of Coping. J Res Spec Educ Needs, 5: 3-10.

McGilloway S., Donnelly M. \& Mays N. (1995) Informal carers of former long-stay hospital residents with learning disabilities. Br J Learn Disabil, 23: 152-5.
Nolan M., Grant G. \& Keady J. (1996) Understanding family care: a multidimensional model of caring and coping. Buckingham, Open University Press.

Olssen M.B. \& Hwang C.P. (2002) Sense of coherence in parents of children with different developmental disabilities. J Intellect Disabil Res, 47: 548-59.

Pearlin L.I., Mullan J.T., Semple S.J. \& Skaff M.M. (1990) Caregiving and the stress process: an overview of concepts and their measures. Gerontologist, 30: 583-91.

Redmond B. \& Richardson V. (2003) Just getting on with it: exploring the service needs of mothers who care for young children with severe/profound and life-threatening intellectual disability. J Appl Res Intellect Disabil, 16: 205-18.

Saloviita T., Italinna M. \& Leinonen E. (2003) Explaining the parental stress of fathers and mothers caring for a child with intellectual disability: a Double ABCX Model. J Intellect Disabil Res, 47: 300-12.

Seltzer M.M., Krauss M.K. Walsh P.N., Conliffe C., Larson B. et al. (1995) Cross-national comparisons of ageing mothers of adults with intellectual disability. J Intellect Disabil Res, 39: 408-18.

Sharry J. (1999) Positively coping: a study of the coping strategies and resourcefulness of parents of a child on the autistic spectrum. Ir J Social Work Res, 2: 87-101.

Shearn J. \& Todd S. (2000) Maternal employment and family responsibilities: the perspective of mothers of children with intellectual disabilities. J Appl Res Intellect Disabil, 13: 109.

Solomon M., Pistrang N. \& Barker C. (2001) The benefits of mutual support groups of parents of children with learning disabilities. Am J Community Psychol, 29: 113-32.

Taanila A., Syrjala L., Kokkonen J. \& Jarvelin M.R. (2002) Coping of parents with physically and/or intellectually disabled children. Child Care Health Dev, 28: 73-86.

Todd S., Shearn J., Beyer S. \& Felce D. (1993) Careers in caring: the changing situation of parents caring for an offspring with learning difficulties. Ir J Psychol, 14: 130-53.

Walsh P.N., Conliffe C. \& Birbeck G. (1993) A study of caregivers of people with intellectual disability in Ireland and Northern Ireland. Ir J Psychol, 14: 176-88.

White N. \& Hastings R.P. (2004) Social and professional support for parents of adolescents with severe intellectual disabilities. J Appl Res Intellect Disabil, 17: 181-90. 